\title{
BMJ Open End-stage renal disease as a risk factor for epiglottitis: a population-based cohort study in Taiwan
}

\author{
Yao-Te Tsai, ${ }^{1}$ Ming-Shao Tsai, ${ }^{1}$ Cheng-Ming Hsu, ${ }^{1}$ Ku-Hao Fang, ${ }^{2}$ Ethan I Huang, ${ }^{1}$ \\ Chia-Yen Liu, ${ }^{3}$ Meng-Hung Lin, ${ }^{3}$ Yao-Hsu Yang, ${ }^{4}$ Yi-Chan Lee, ${ }^{5}$ \\ Geng-He Chang (D) ${ }^{1}$
}

To cite: Tsai Y-T, Tsai M-S, Hsu C-M, et al. End-stage renal disease as a risk factor for epiglottitis: a population-based cohort study in Taiwan. BMJ Open 2020;10:e038683. doi:10.1136/ bmjopen-2020-038683

- Prepublication history for this paper is available online. To view these files, please visit the journal online (http://dx.doi. org/10.1136/bmjopen-2020038683).

Received 23 March 2020 Revised 15 July 2020 Accepted 08 October 2020

Check for updates

(c) Author(s) (or their employer(s)) 2020. Re-use permitted under CC BY-NC. No commercial re-use. See rights and permissions. Published by BMJ.

${ }^{1}$ Department of Otolaryngology Head and Neck Surgery, Chang Gung Memorial Hospital, Chiayi Branch, Puzi, Taiwan

${ }^{2}$ Department of Otolaryngology Head and Neck Surgery, Chang Gung Memorial Hospital,

Taoyuan, Taiwan

${ }^{3}$ Health Information and Epidemiology Laboratory, Chang Gung Memorial Hospital, Chiayi Branch, Puzi, Taiwan

${ }^{4}$ Department of Traditional Chinese Medicine, Chang Gung Memorial Hospital, Chiayi

Branch, Puzi, Taiwan

${ }^{5}$ Department of Otolaryngology Head and Neck Surgery, Chang Gung Memorial Hospital, Keelung, Taiwan

Correspondence to Dr Geng-He Chang; trance881057@gmail.com

\section{ABSTRACT}

Objectives Patients with uremia are prone to infection; however, end-stage renal disease (ESRD) as a risk factor for acute epiglottitis warrants study. We investigated the risk of severe epiglottitis requiring hospitalisation in patients with ESRD.

Setting We conducted a retrospective matched cohort study by using the claims data of Taiwan's National Health Insurance Research Database.

Participants We identified an ESRD cohort with 87908 patients newly diagnosed in 2000-2013 and underwent dialysis. The non-ESRD cohort comprised patients who had not received a diagnosis of ESRD, and they were matches to the ESRD cohort (1:1) by sex, age, residence urbanisation level, monthly income, and diabetes and hypertension status.

Primary and secondary outcome measures The cumulative incidence of epiglottitis at the end of 2013 was analysed with Kaplan-Meier methods and log-rank tests. The HR of epiglottitis was calculated using the Cox proportional hazards model after adjustment for confounding factors.

Results The overall epiglottitis incidence rate was $94 \%$ greater in the ESRD cohort than in the non-ESRD cohort (10.3 vs 5.3 cases per 100000 person-years, $p=0.002$ ), with an adjusted HR of 1.89 (95\% Cl: 1.23 to 2.91, $\mathrm{p}=0.004)$. In the log-rank analysis, compared with the non-ESRD group, the epiglottitis cumulative incidence was significantly higher in the ESRD group ( $p=0.003$ ). Epiglottitis did not exhibit an association with higher rates of airway interventions, intensive care unit admissions or longer hospitalisation in patients with ESRD than in controls.

Conclusions This nationwide matched cohort study indicated that ESRD patients should be monitored for the risk of severe epiglottitis requiring hospitalisation.

\section{INTRODUCTION}

Epiglottitis is characterised by acute supraglottic inflammatory swelling and has a high risk of airway obstruction if not appropriately managed. Epiglottitis is most commonly caused by bacteria; other causes include trauma by a foreign object, viral or fungal infections, chemical ingestion and inhalation burns. ${ }^{1}$ Introducing the Haemophilus
Strength and limitations of this study

- We conducted this population-based retrospective cohort study by using the National Health Insurance Research Database (NHIRD) in Taiwan.

- This is the first large-scale study to investigate the risk of acute epiglottitis in patients with end-stage renal disease over a long-term follow-up period.

- The long-term follow-up and large sample size of the present study provided substantial statistical power.

- The NHIRD did not provide some details (eg, smoking habits, results of image study or blood tests for the severity levels of infection); therefore, we could not account for these potential confounding factors in the analysis.

- This study can reflect the real-world conditions in Taiwan, and it is more generalisable than studies using tertiary referral centre databases.

influenzae type-b (Hib) vaccine to programmes of childhood vaccination subsequently engendered a considerable decline in the incidence of acute epiglottitis among children; nevertheless, the incidence increased among adults. ${ }^{23}$ Epiglottitis might present a distinct scenario in adults. In children, an acute onset of symptoms typically appears in formerly healthy patients. However, up to $50 \%$ of adults with epiglottitis have comorbidities such as alcohol abuse, hypertension (HTN) and diabetes mellitus (DM) at diagnosis; such comorbidities could impair patients' immune systems as well as raise their proneness to multiple infections. ${ }^{2}{ }^{4-7}$ By retrospectively analysing the data of 6072 adult patients with epiglottitis derived from Japan's national database, Suzuki et aldemonstrated that patients who had underlying chronic renal failure, DM, epiglottic cysts and were older had more severe epiglottitis and complications. ${ }^{8}$ Ischaemic heart disease, uremia, non-haematologic malignancy and 
other systemic diseases are also risk factors for severe epiglottitis that requires hospitalisation. ${ }^{18}$

Although epiglottitis is a severe inflammatory disease, it may also occur in patients with compromised immune systems or impaired inflammatory responses. End-stage renal disease (ESRD) is prevalent globally; in addition, Taiwan has the highest renal replacement therapy rate in the world because the implementation of the National Health Insurance (NHI) programme increased the number of people receiving ESRD treatment since the 1990s. ${ }^{9}$ ESRD is associated with immunocompromised status and significantly increases morbidity and mortality resulting from infections, which may account for $20 \%$ of total mortality in patients with ESRD. ${ }^{10}$ Although chronic renal failure increases susceptibility to epiglottitis, whether epiglottitis is more frequent or has a different clinical course in patients with ESRD remains unknown. ${ }^{8}$ On the basis of these research findings, an association between ESRD and epiglottitis can be hypothesised. Nevertheless, a lack of relevant evidence prevents the formulation of salutary advices regarding clinical practice. To gain a comprehensive understanding of epiglottitis risk and prognosis in patients with ESRD, we used data from Taiwan's National Health Insurance Research Database (NHIRD) to test our hypothesis. In particular, we conducted this retrospective population-based cohort study to probe the incidence and risk of epiglottitis with hospitalisation (primary endpoints) along with hospitalisation length, airway interventions, intensive care unit (ICU) admission and survival outcomes (secondary endpoints).

\section{MATERIALS AND METHODS \\ Data source}

In March 1995, Taiwan's government implemented the mandatory NHI programme. Since its implementation, the programme has provided medical service coverage for over 99\% of Taiwan's 23.5 million residents; disease diagnoses in the programme are made on the basis of International Classification of Diseases, Ninth Revision, Clinical Modification (ICD-9-CM) diagnostic codes. ${ }^{11}$ The NHIRD, the programme's database, comprises comprehensive personal information, prescription details and diagnostic codes as well as data on clinic visits and surgical procedures for all insurants. NHIRD data are encrypted to ensure patient privacy.

\section{Patient and public involvement}

Patients were not involved in the design or execution of this study.

\section{Study design and participants}

Patients in this study were assigned to an ESRD or control group, as presented by the flowchart in figure 1 . In the NHI system, 'catastrophic illness patient (CIP)' refers to a patient who may benefit from treatment for catastrophic disorders. Verifying CIP eligibility is governed by strict criteria, and patients with CIP status in the NHIRD are enrolled in the Registry for Catastrophic Illness Patients (RFCIP). The ESRD group in this study comprised patients in the RFCIP who were recorded as receiving a new diagnosis of ESRD (ICD-9-CM: 585) and as receiving dialysis between 1 January 2000, and 31 December 2013. ${ }^{12}$ Patients determined to have missing data, to be younger than 18 years or to have received a diagnosis of a preexisting malignancy (ICD-9-CM: 140-208) were excluded from this study. Moreover, patients diagnosed as having epiglottitis before receiving an ESRD diagnosis were excluded to ensure result validity regarding the susceptibility of patients with ESRD to developing epiglottitis. A total of 87908 patients constituted the ESRD cohort.

The Longitudinal Health Insurance Database 2000 (LHID2000) represents approximately 5\% of Taiwan's residents and includes the medical claims data (19962013) of 1 million individuals who were selected at random through systematic sampling from the 2000 Registry for Beneficiaries of the NHIRD. As reported by the Taiwan National Health Research Institutes, LHID2000 enrollees do not differ significantly from all NHI programme enrollees in terms of healthcare costs, sex or age. ${ }^{13}$ We formed the control group by matching each ESRD case with one randomly selected control without ESRD (1:1) from the LHID2000 in terms of monthly income, age, residence urbanisation level, DM, sex and HTN. Because DM and HTN are notable covariates of epiglottitis, we matched patients with these diseases in this process. ${ }^{46-8}$

For the ESRD group, the index date was the RFCIP registration date. The index dates of patients who constituted the control group were matched with the dates of those who constituted the ESRD group. Patients were excluded if they received a diagnosis of epiglottitis before the index date. We finally executed our follow-up study on 80636 ESRD patients with dialysis and 80636 matching controls. We executed patient follow-up until NHI withdrawal, the end of the study period (31 December 2013) or death. The period spanning from the index date to epiglottitis diagnosis constituted the follow-up period; because of death, some patients were censored.

\section{Outcome and covariate measurements}

Severe epiglottitis in adults was the primary outcome (ICD-9-CM: $464.3,464.30$ or 464.31 ). Severity was defined as a diagnosis of acute epiglottitis confirmed by otolaryngologists and necessitating acute care hospital admission. Therefore, to determine the incidence of severe epiglottitis in each cohort, we selected patients 18 years or older who were first diagnosed as having epiglottitis during hospitalisation without concomitant deep neck infection, namely parapharyngeal abscess (ICD-9-CM: 478.22), cellulitis and abscess of neck (ICD-9-CM: 682.11), retropharyngeal abscess (ICD-9-CM: 478.24) or cellulitis and abscess of oral soft tissues (ICD-9-CM: 528.3). ${ }^{11}$ For both groups, epiglottitis diagnostic codes were used to determine epiglottitis incidence until 31 December 2013. From inpatient and ambulatory claims data, we 


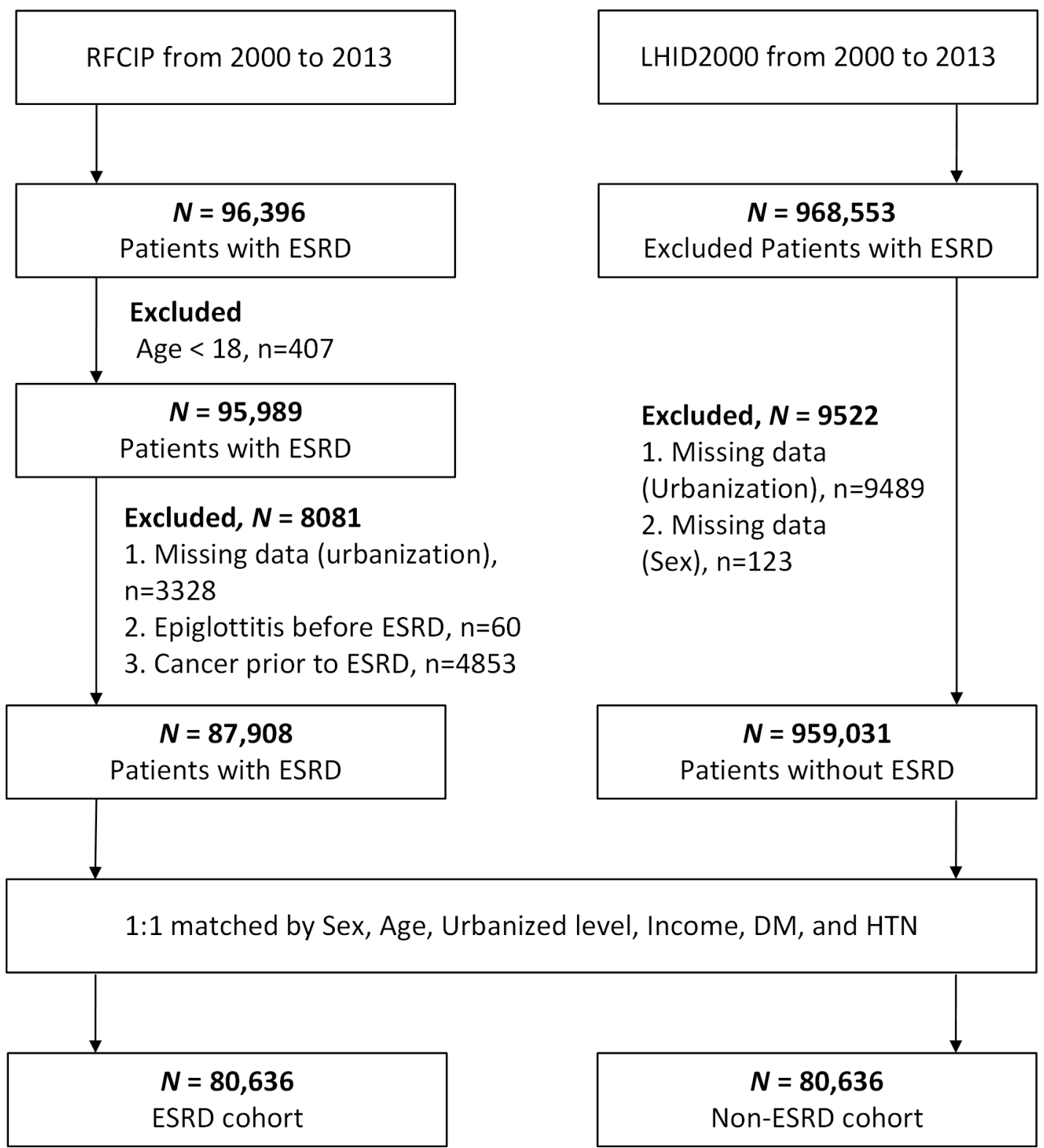

Figure 1 Enrolment schema of the ESRD and control cohorts. Patients with ESRD were identified between 2000 and 2013 in Taiwan from the RFCIP database, and a total of 96396 patients were included. A total of 407 patients aged less than 18 years, 3328 patients with missed urbanisation data, 60 patients with epiglottitis occurring prior to the index date of ESRD and 4854 patients with a history of malignancy prior to the index date of ESRD were all excluded. A total of 87908 patients with ESRD were eligible and included in the ESRD cohort. The LHID2000 consisting of 968553 insurants representing the general population in Taiwan was used to match with the ESRD cohort for urbanisation level, income level, age, sex, DM and HTN with 1:1 fusion. Finally, the matched cohort study was conducted on 80636 patients with ESRD (the ESRD group) and 80636 patients without ESRD (the control group). DM, diabetes mellitus; ESRD, end-stage renal disease; HTN, hypertension; LHID2000, Longitudinal Health Insurance Database 2000; RFCIP, Registry for Catastrophic IIIness Patients.

retrieved information on the following covariates related to epiglottitis: DM (ICD-9-CM: 250.xx), chronic obstructive pulmonary disease (COPD; ICD-9-CM: 491, 492 and 496), liver cirrhosis (ICD-9-CM: 571.2 and 571.5-571.6), HTN (ICD-9-CM: 401-405) and gastroesophageal reflux disease (GERD; ICD-9-CM: 530.11, 530.81 and 530.85). Covariates that occurred within 12 months of the index date and appeared at least once in inpatient claims or at least three times in ambulatory care claims were included. To evaluate prognoses, we also analysed hospitalisation length, ICU admission and number of patients receiving intubation and tracheostomy surgery. Mortality defined as death during epiglottitis treatment or within 1 month after epiglottitis diagnosis was analysed in both cohorts.

\section{Statistical analysis}

To compare the groups in terms of sociodemographic characteristics and covariates, Student's independent t-test and Pearson's $\chi^{2}$ test were used to analyse continuous and descriptive variables, respectively. We derived 
Table 1 Demographic characteristics of the ESRD and control groups

\begin{tabular}{|c|c|c|c|}
\hline \multirow[b]{2}{*}{ Variable } & \multicolumn{3}{|c|}{ Number (\%) of individuals } \\
\hline & ESRD (n=80 636) & Non-ESRD (n=80 636) & P value* \\
\hline Sex & & & 1.00 \\
\hline Men & $40562(50.3)$ & $40562(50.3)$ & \\
\hline Women & $40074(49.7)$ & $40074(49.7)$ & \\
\hline Age (years) & & & 1.00 \\
\hline $18-39$ & $6197(7.7)$ & $6197(7.7)$ & \\
\hline $40-64$ & $40000(49.6)$ & $40000(49.6)$ & \\
\hline$\geq 65$ & $34439(42.7)$ & $34439(42.7)$ & \\
\hline Monthly income (NTD) & & & 1.00 \\
\hline 0 & $16274(20.2)$ & 16274 (20.2) & \\
\hline $1-15840$ & $12634(15.7)$ & $12634(15.7)$ & \\
\hline $15841-25000$ & $39185(48.6)$ & $39185(48.6)$ & \\
\hline$\geq 25001$ & $12543(15.6)$ & $12543(15.6)$ & \\
\hline Urbanisation level & & & 1.00 \\
\hline 1 (city) & $22149(27.5)$ & $22149(27.5)$ & \\
\hline 2 & $37151(46.1)$ & $37151(46.1)$ & \\
\hline 3 & $14016(17.4)$ & $14016(17.4)$ & \\
\hline 4 (village) & $7320(9.1)$ & $7320(9.1)$ & \\
\hline Epiglottitis & $52(0.06)$ & $35(0.04)$ & 0.06 \\
\hline \multicolumn{4}{|l|}{ Covariates } \\
\hline Chronic liver disease & $23737(29.4)$ & $22584(28.0)$ & $<0.01$ \\
\hline HTN & $77433(96.0)$ & 77433 (96.0) & 1.00 \\
\hline COPD & $29934(37.1)$ & $28122(34.9)$ & $<0.01$ \\
\hline GERD & $14513(18.0)$ & $9806(12.2)$ & $<0.01$ \\
\hline DM & $52346(64.9)$ & $52346(64.9)$ & 1.00 \\
\hline
\end{tabular}

*Pearson's $\chi^{2}$ tests.

COPD, chronic obstructive pulmonary disease; DM, diabetes mellitus; ESRD, end-stage renal disease; GERD, gastroesophageal reflux disease; HTN, hypertension ; NTD, New Taiwan dollar.

incidence rate by dividing the number of epiglottitis diagnoses in the follow-up period by the total personyears for each group according to length of follow-up. In both groups, the cumulative incidence of epiglottitis was derived using Kaplan-Meier analysis. We also used a Cox proportional hazards model to estimate the epiglottitis risk in the ESRD group. We executed Student's t-test to conduct comparisons for hospitalisation length and executed Fisher's exact test to perform comparisons for tracheostomy rate. By employing Pearson's $\chi^{2}$ test, we conducted comparisons for the numbers of patients requiring intubation and ICU admission. SAS V.9.4 was executed to conduct the entirety of this study's statistical analyses, with a two-sided $p$ value of $<0.05$ being deemed as representing statistical significance.

\section{RESULTS}

The observed distributions of sociodemographic characteristics along with epiglottitis and covariates in the
ESRD and control cohorts are presented in table 1. After matching by age, sex, socioeconomic status, DM and HTN, we determined the ESRD group to be more prone to covariates, namely, COPD, GERD and liver cirrhosis, when compared with the control group (table 1). Among the 80636 patients with ESRD, $52(0.06 \%)$ had epiglottitis, and among the 80636 controls, $35(0.04 \%)$ had epiglottitis. The mean (SD) follow-up duration for the ESRD group was 6.2 years (3.8 years), and that for the control group was 8.2 years (3.6 years).

Figure 2 presents the cumulative incidence of epiglottitis, as derived from the Kaplan-Meier analysis, in both cohorts during 2000-2013. In a log-rank analysis, compared with the control group, the epiglottitis cumulative incidence was determined to be significantly higher in the ESRD group ( $p=0.003$; figure 2). A 1.94-fold higher epiglottitis incidence rate was observed in the ESRD group relative to the control group (10.3 vs 5.3 cases per 100000 person-years; table 2). A stratified analysis of the 

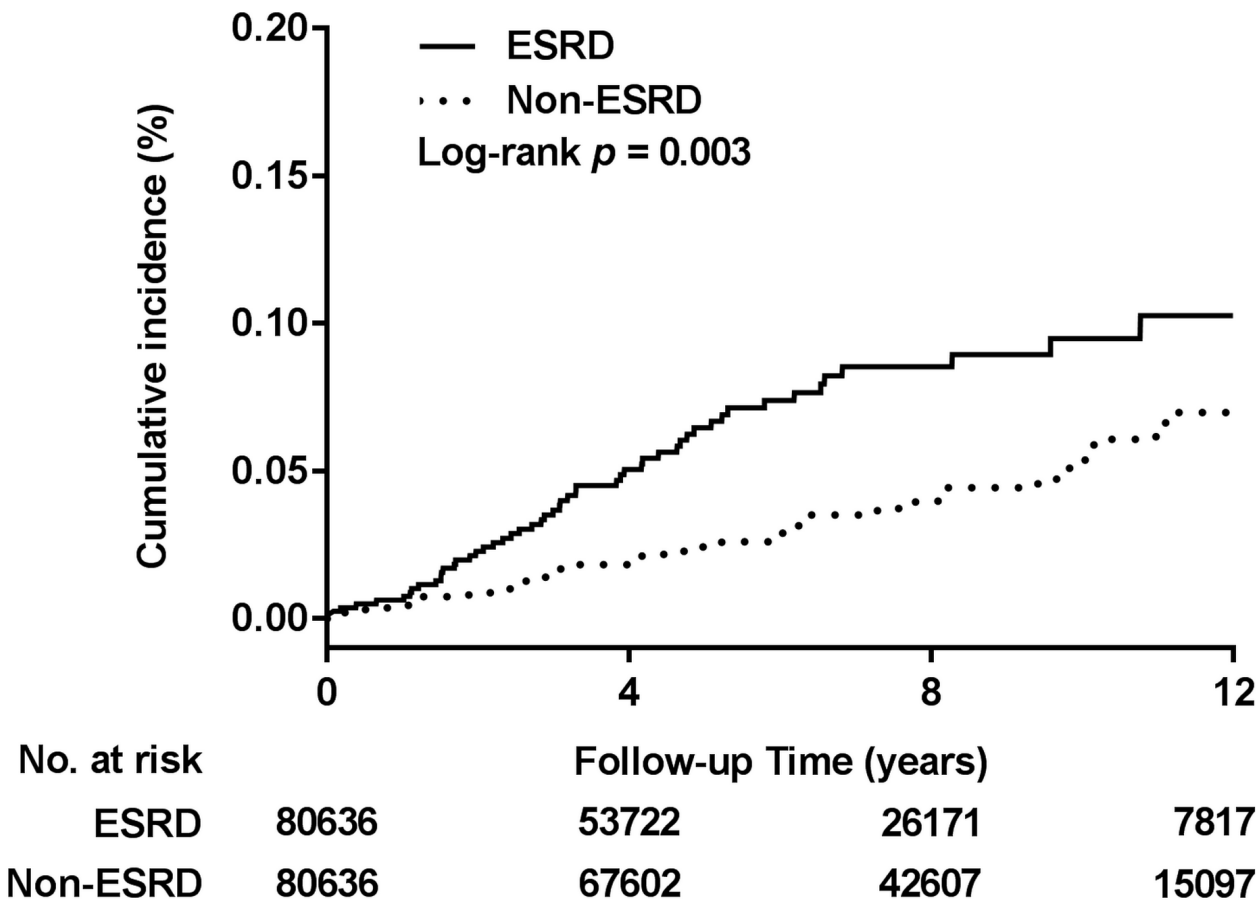

Follow-up Time (years)

$\begin{array}{rrr}53722 & 26171 & 7817 \\ 67602 & 42607 & 15097\end{array}$

Figure 2 Cumulative incidence of epiglottitis for end-stage renal disease (ESRD) versus non-ESRD. The Kaplan-Meier analysis demonstrated cumulative epiglottitis identified in the ESRD and control cohorts, respectively, during the follow-up period (20002013). The log-rank test revealed a significantly higher cumulative incidence in the ESRD group ( $p=0.003)$.

follow-up period revealed a significantly higher incidence of epiglottitis in the ESRD group than in the control group when the groups were stratified by 1-5 years of follow-up $(\mathrm{p}<0.01$; table 2$)$.

We applied the aforementioned Cox proportional hazards model to execute crude and adjusted HR (aHR) analyses for both groups after adjustment for residence urbanisation level, sex, selected covariates, monthly income and age (table 3). We noted the ESRD group to have a 1.89-fold higher risk of epiglottitis relative to the control group (aHR: 1.89, 95\% CI: 1.23 to 2.91, $\mathrm{p}<0.01$; table 3). In the Cox regression analysis, the effects of age, sex and the underlying covariates of COPD, DM, GERD, HTN and liver cirrhosis on epiglottitis were statistically non-significant.

Table 4 lists results derived in this study regarding the airway interventions (intubation and tracheostomy) used to treat epiglottitis, hospitalisation duration, ICU admission and mortality in both groups. Although more patients with ESRD required intubation (ESRD group: $17.3 \%$; control group: $14.3 \%$; $\mathrm{p}=0.71$ ), tracheostomy (ESRD group: $7.7 \%$; control group: $2.9 \%$; $\mathrm{p}=0.64$ ) and ICU admission (ESRD group: 44.2\%; control group: $25.7 \%$; $\mathrm{p}=0.07$ ), this trend did not reach statistical significance. Moreover, hospitalisation duration (ESRD group: $9.9 \pm 9.8$ days; control group: $7.9 \pm 11.6$ days; $\mathrm{p}=0.37$ ) was not significantly different between these groups. The ESRD group was also noted to have a higher mortality rate 1 month after epiglottitis diagnosis than the controls did because no mortality was observed in the patients without ESRD.

\section{DISCUSSION}

According to our literature review, this study is the first to analyse epiglottitis risk in patients with ESRD. The

Table 2 Risk of epiglottitis in the ESRD and control group

\begin{tabular}{|c|c|c|c|c|c|c|c|c|c|c|}
\hline & \multicolumn{4}{|l|}{ ESRD } & \multicolumn{4}{|c|}{ Controls } & \multirow[b]{2}{*}{ IRR (95\% CI) } & \multirow[b]{2}{*}{$P$ value } \\
\hline & $\mathbf{N}$ & Epiglottitis & PYs & Rate & $\mathbf{N}$ & Epiglottitis & PYs & Rate* $^{*}$ & & \\
\hline Overall & 80636 & 52 & 503377.8 & 10.3 & 80636 & 35 & 657428.1 & 5.3 & 1.94 (1.26 to 2.98$)$ & $<0.01$ \\
\hline$<1$ & 80636 & 5 & 78645.2 & 6.4 & 80636 & 3 & 80223.4 & 3.7 & $1.70(0.41$ to 7.11$)$ & 0.46 \\
\hline $1-5$ & 75714 & 36 & 244619.1 & 14.7 & 79561 & 15 & 287934.1 & 5.2 & 2.83 (1.55 to 5.16$)$ & $<0.01$ \\
\hline
\end{tabular}

*Per 100000 PYs; IRR was compared using Poisson regression.

ESRD, end-stage renal disease; PYs, person-years; IRR, incidence rate ratio. 
Table 3 Multivariable Cox proportional hazard regression of the association between epiglottitis and potential risk factors

\begin{tabular}{|c|c|c|c|c|}
\hline Variables & Crude HR (95\% CI) & $P$ value & Adjusted HR $(95 \% \mathrm{Cl})^{*}$ & $P$ value \\
\hline \multicolumn{5}{|l|}{ Exposure } \\
\hline Non-ESRD & 1 & & 1 & \\
\hline ESRD & 1.90 (1.23 to 2.92$)$ & $<0.01$ & 1.89 (1.23 to 2.91$)$ & $<0.01$ \\
\hline \multicolumn{5}{|l|}{ Sex } \\
\hline Women & 1 & & 1 & \\
\hline Men & 1.34 (0.88 to 2.05$)$ & 0.17 & 1.38 (0.89 to 2.14$)$ & 0.15 \\
\hline \multicolumn{5}{|l|}{ Age (years) } \\
\hline 18-39 & 1 & & 1 & \\
\hline $40-64$ & 0.75 (0.39 to 1.44$)$ & 0.38 & 0.91 (0.45 to 1.81$)$ & 0.77 \\
\hline$\geq 65$ & 0.55 (0.27 to 1.11$)$ & 0.09 & 0.59 (0.28 to 1.23$)$ & 0.15 \\
\hline \multicolumn{5}{|l|}{ Covariates } \\
\hline COPD & 1.27 (0.83 to 1.95$)$ & 0.26 & 1.40 (0.90 to 2.19$)$ & 0.13 \\
\hline $\mathrm{DM}$ & 0.76 (0.50 to 1.17$)$ & 0.21 & 0.82 (0.52 to 1.27$)$ & 0.36 \\
\hline HTN & 0.70 (0.36 to 1.36$)$ & 0.29 & 0.61 (0.32 to 1.19$)$ & 0.15 \\
\hline GERD & 0.70 (0.29 to 1.74$)$ & 0.44 & 0.77 (0.31 to 1.92$)$ & 0.57 \\
\hline Liver cirrhosis & 1.31 (0.84 to 2.04$)$ & 0.23 & $1.29(0.82$ to 2.01$)$ & 0.27 \\
\hline
\end{tabular}

${ }^{*}$ Cox model are adjusted by sex, age, monthly income, urbanised level and covariates.

COPD, chronic obstructive pulmonary disease; DM, diabetes mellitus; ESRD, end-stage renal disease; GERD, gastro-oesophageal reflux disease; HTN, hypertension .

long observation periods $(6.2 \pm 3.8$ years and $8.2 \pm 3.6$ years in the ESRD and control groups, respectively) were adequate for observing differences in epiglottitis risk between the groups. The overall epiglottitis incidence rate was $94 \%$ higher in the ESRD cohort than in the nonESRD cohort (10.3 vs 5.3 cases per 100000 person-years, $\mathrm{p}=0.002$ ), and the log-rank test revealed that the cumulative incidence of epiglottitis was significantly higher in

\begin{tabular}{|c|c|c|c|}
\hline \multirow[t]{2}{*}{ Characteristic } & \multicolumn{2}{|l|}{ Number (\%) } & \multirow[b]{2}{*}{$\begin{array}{l}P \\
\text { value }\end{array}$} \\
\hline & ESRD-epiglottitis & $\begin{array}{l}\text { Control- } \\
\text { epiglottitis }\end{array}$ & \\
\hline Total & 52 & 35 & \\
\hline \multicolumn{4}{|l|}{$\begin{array}{l}\text { Airway } \\
\text { intervention }\end{array}$} \\
\hline Intubation & $9(17.3)$ & $5(14.3)$ & $0.71^{*}$ \\
\hline Tracheostomy & $4(7.7)$ & $1(2.9)$ & $0.64 \dagger$ \\
\hline $\begin{array}{l}\text { Hospitalisation } \\
(\text { mean } \pm S D)\end{array}$ & $9.92 \pm 9.91$ & $7.9 \pm 11.6$ & $0.37 \ddagger$ \\
\hline ICU care & 23 (44.2) & $9(25.7)$ & $0.07^{*}$ \\
\hline Mortality§ & $2(3.9)$ & $0(0)$ & NA \\
\hline
\end{tabular}

*Pearson's $\chi^{2}$ tests.

†Fisher's exact test.

¥Student's t-test.

§Mortality occurrence within 1 month after epiglottitis.

ESRD, end-stage renal disease; ICU, intensive care unit; NA, not applicable. the ESRD group ( $p=0.003)$. We controlled the effects of potential confounders by using aHRs (after adjustment for COPD, GERD, DM, HTN and liver cirrhosis) in our outcome comparisons, and derived results confirmed that ESRD was an independent epiglottitis risk factor with approximately two times higher risk than that in the non-ESRD cohorts. In addition, epiglottitis was association with higher rates of airway interventions, ICU admissions and longer hospitalisation in patients with ESRD than in controls. However, the results did not reach statistical significance probably because of the relatively small sample size. In reality, collecting sufficient samples with an adequate follow-up duration at a single medical institution to investigate long-term epiglottitis incidence after ESRD diagnosis would be difficult. By using Taiwan's NHIRD, we traced the majority of ESRD and epiglottitis cases and minimised selection bias since the NHI programme covers nearly all medical services. Our findings support our hypothesis and demonstrate that ESRD is an independent risk factor for adult epiglottitis.

Patients with ESRD are particularly susceptible to infections, which cause morbidity and are second to cardiovascular events in causing death in patients receiving haemodialysis. ${ }^{14}$ In this population, bacteraemia, peritonitis, influenza, tuberculosis and pneumonia are responsible for most severe infections. ${ }^{15}$ Of patients with acute epiglottis, $0.5 \%-9 \%$ have comorbid renal impairment. ${ }^{816}$ However, the association between ESRD and epiglottitis lacked verification because the disease is rare and control participants to test for statistical significance were lacking. Factors possibly explaining the significant 
ESRD-epiglottitis association that we observed are altered innate immunity resulting from azotemia, reduced expression of monocyte Toll-like receptor $4,{ }^{17}$ impaired polymorphonuclear chemotaxis and phagocytosis, ${ }^{18}$ and diffuse reduction of B-lymphocyte cells ${ }^{19}$ that increase ESRD patients' likelihood to develop acute epiglottitis. Moreover, Streptococcus pneumoniae causes most ESRDassociated respiratory tract infections ${ }^{20}$ and is the most critical bacterial cause of acute epiglottitis in vaccinated children ${ }^{21}$ as well as adults. ${ }^{22}$ Hence, we can assume that adult epiglottitis and common respiratory infection in patients with ESRD share the same pathogen, which may increase the risk of epiglottitis among patients with ESRD. Future studies may investigate the efficacy of the group $\mathrm{B}$ streptococcus vaccination in preventing epiglottitis in patients with ESRD. These findings suggest that ESRD affects the development of acute epiglottitis, rendering ESRD an independent risk factor.

Among patients with epiglottitis, up to $62 \%$ have been reported to be admitted to the ICU, with $7 \%-30 \%$ receiving airway interventions, including tracheostomy $(3.5 \%-7.7 \%)$ and intubation $(5.6 \%-26 \%) .{ }^{478}$ Our results reveal that the airway intervention rate after epiglottitis diagnosis in the ESRD group was not significantly greater than that in the control group. The airway intervention rates observed for the two cohorts are consistent with those reported in previous research. We also observed that $36 \%$ of patients with epiglottitis were admitted to the ICU, $71.8 \%$ of whom had ESRD. The ICU admission rates in both cohorts are also consistent with those $(28.3 \%-51 \%)$ in the literature. ${ }^{421}$ However, patients with ESRD did not have longer hospital stays or higher ICU admission rates than the controls did in this study. Furthermore, patients with ESRD in our study had a higher mortality rate after epiglottitis because no deaths occurred in the control cohort. Therefore, our findings suggest that epiglottitis in patients with ESRD is not considerably associated with an increased risk of airway interventions or complicated clinical courses; however, the higher rates of airway interventions and ICU admission in the ESRD cohort may partially explain the higher mortality rate. The present study results can offer a complete spectrum of treatment and prognosis for epiglottitis and are consistent with the results of a relevant study on head and neck infections that indicated that patients with ESRD and deep neck infections did not have higher rates of airway interventions or longer hospital stays than patients without ESRD. ${ }^{11}$ This may have occurred because the prognosis of acute epiglottitis is typically positive, even in critically ill patients requiring ICU admission, if appropriate treatment and timely airway intervention are performed. ${ }^{416}$ In addition, the clinical effect of ESRD on epiglottitis treatment may not have reached statistical significance because of the limited sample size in the present study.

Our current study is the first to probe the epiglottitis-ESRD relationship. Nevertheless, the incidence of epiglottitis in Taiwan is higher (similar to that in Finland) compared with that in other retrospective studies on epiglottitis in adults. ${ }^{412324}$ Because Hib vaccination began in Taiwan in 1996, we can assume that the vaccine did not affect adult epiglottitis incidence in our study. The increased incidence has no direct explanation, but the prevalent use of flexible nasopharyngeal fibrescopes and the high accessibility of Taiwan's healthcare system may account for the increased incidence of epiglottitis during the study period to some degree because both phenomena enhance diagnostic sensitivity. Our study has a few limitations. First, to evaluate prognostic outcomes, ICD-9-CM codes were used in this study to determine patients with epiglottitis as well as the corresponding clinical courses and airway interventions in both cohorts; however, the claims' data did not have details on epiglottitis severity, such as laboratory tests, cultured pathogen or epiglottitis-related imaging findings. Therefore, we could not evaluate the association between epiglottitis severity and ESRD. Second, because patients with ESRD visit the hospital more frequently than does the general population, surveillance bias may have resulted because patients with ESRD have more access to otolaryngologists for flexible nasopharyngolaryngoscopy examinations. Although our results reach statistical significance regarding ESRD as a risk factor for epiglottitis, they should be interpreted while considering these limitations.

\section{CONCLUSION}

This nationwide population-based study analysed epidemiological data on acute epiglottitis and its prognosis in patients with ESRD. Our findings demonstrate ESRD to constitute an independent risk factor for adult epiglottitis. Epiglottitis was associated with higher rates of airway interventions, ICU admissions and longer hospitalisation in patients with ESRD than in controls; nevertheless, the results did not reach statistical significance. Additional large-scale, prospective studies are warranted to confirm our findings.

Contributors Y-TT and M-ST conceived of the study, participated in its design and coordination, data interpretation, performed the statistical analysis and drafted the manuscript. C-MH, K-HF, ElH, Y-CL and Y-HY participated in the design of the study and data interpretation. $\mathrm{C}-\mathrm{YL}$ and $\mathrm{M}-\mathrm{HL}$ participated in the statistical analysis and data interpretation. Y-TT and G-HC wrote the paper. All authors have read and approved the final manuscript.

Funding This work was supported by the grant (CFRPG6J0071) and (CMRPG6J0201) from Chang Gung Memorial Hospital, Taiwan.

Competing interests None declared.

Patient consent for publication Our use of anonymous data obviated the necessity of informed consent, as waived by the Chang Gung Memorial Hospital's Institutional Review Board.

Ethics approval Our executed study protocol was ratified by Chang Gung Memorial Hospital's Institutional Review Board (No. 201801786B0C501).

Provenance and peer review Not commissioned; externally peer reviewed.

Data availability statement No additional data are available.

Open access This is an open access article distributed in accordance with the Creative Commons Attribution Non Commercial (CC BY-NC 4.0) license, which permits others to distribute, remix, adapt, build upon this work non-commercially, 
and license their derivative works on different terms, provided the original work is properly cited, appropriate credit is given, any changes made indicated, and the use is non-commercial. See: http://creativecommons.org/licenses/by-nc/4.0/.

ORCID iD

Geng-He Chang http://orcid.org/0000-0001-5939-9747

\section{REFERENCES}

1 Tsai Y-T, Huang El, Chang G-H, et al. Risk of acute epiglottitis in patients with preexisting diabetes mellitus: a population-based casecontrol study. PLoS One 2018;13:e0199036.

2 Shah RK, Stocks C. Epiglottitis in the United States: national trends, variances, prognosis, and management. Laryngoscope 2010;120:1256-62.

3 Wood N, Menzies R, Mclntyre P. Epiglottitis in Sydney before and after the introduction of vaccination against Haemophilus influenzae type B disease. Intern Med J 2005;35:530-5.

4 Bizaki AJ, Numminen J, Vasama J-P, et al. Acute supraglottitis in adults in Finland: review and analysis of 308 cases. Laryngoscope 2011;121:2107-13.

5 Frantz TD, Rasgon BM, Quesenberry CP. Acute epiglottitis in adults. Analysis of 129 cases. JAMA 1994;272:1358-60.

6 Mayo-Smith MF, Spinale JW, Donskey CJ, et al. Acute epiglottitis. An 18-year experience in Rhode island. Chest 1995;108:1640-7.

$7 \mathrm{HL} \mathrm{N}$, Sin LM, MF L, et al. Acute epiglottitis in adults: a retrospective review of 106 patients in Hong Kong. Emerg Med J 2008;25:253-5.

8 Suzuki S, Yasunaga $\mathrm{H}$, Matsui $\mathrm{H}$, et al. Factors associated with severe epiglottitis in adults: analysis of a Japanese inpatient database. Laryngoscope 2015;125:2072-8.

9 Yang W-C, Hwang S-J, Taiwan Society of Nephrology. Incidence, prevalence and mortality trends of dialysis end-stage renal disease in Taiwan from 1990 to 2001: the impact of national health insurance. Nephrol Dial Transplant 2008;23:3977-82.

10 Kato S, Chmielewski M, Honda $\mathrm{H}$, et al. Aspects of immune dysfunction in end-stage renal disease. Clin J Am Soc Nephrol 2008;3:1526-33.

11 Chang G-H, Tsai M-S, Liu C-Y, et al. End-stage renal disease: a risk factor of deep neck infection - a nationwide follow-up study in Taiwan. BMC Infect Dis 2017;17:424.
12 Wu P-P, Kor C-T, Hsieh M-C, et al. Association between endstage renal disease and incident diabetes Mellitus-A nationwide population-based cohort study. J Clin Med 2018;7. doi:10.3390/ jcm7100343. [Epub ahead of print: 1110 2018].

13 Yang $\mathrm{Y}-\mathrm{H}$, Chen W-C, Tsan Y-T, et al. Statin use and the risk of cirrhosis development in patients with hepatitis $C$ virus infection. $J$ Hepatol 2015;63:1111-7.

14 Collier S, Davenport A. Reducing the risk of infection in end-stage kidney failure patients treated by dialysis. Nephrol Dial Transplant 2014;29:2158-61.

15 Wakasugi M, Kawamura K, Yamamoto S, et al. High mortality rate of infectious diseases in dialysis patients: a comparison with the general population in Japan. Ther Apher Dial 2012;16:226-31.

16 Chroboczek T, Cour M, Hernu R, et al. Long-Term outcome of critically ill adult patients with acute epiglottitis. PLoS One 2015;10:e0125736.

17 Koc M, Toprak A, Arikan H, et al. Toll-Like receptor expression in monocytes in patients with chronic kidney disease and haemodialysis: relation with inflammation. Nephrol Dial Transplant 2011;26:955-63.

18 Cohen G, Haag-Weber M, Hörl WH. Immune dysfunction in uremia. Kidney Int Suppl 1997;62:S79-82.

19 Pahl MV, Gollapudi S, Sepassi L, et al. Effect of end-stage renal disease on B-lymphocyte subpopulations, IL-7, BAFF and BAFF receptor expression. Nephrol Dial Transplant 2010;25:205-12.

20 Eleftheriadis T, Liakopoulos V, Leivaditis K, et al. Infections in hemodialysis: a concise review - Part 1: bacteremia and respiratory infections. Hippokratia 2011;15:12-17.

21 Briem B, Thorvardsson O, Petersen H. Acute epiglottitis in Iceland 1983-2005. Auris Nasus Larynx 2009;36:46-52.

22 Isakson M, Hugosson S. Acute epiglottitis: epidemiology and Streptococcus pneumoniae serotype distribution in adults. $J$ Laryngol Otol 2011;125:390-3.

23 Hébert PC, Ducic Y, Boisvert D, et al. Adult epiglottitis in a Canadian setting. Laryngoscope 1998;108:64-9.

24 Price IM, Preyra I, Fernandes CMB, et al. Adult epiglottitis: a fiveyear retrospective chart review in a major urban centre. CJEM 2005; 7:387-90. 